\title{
Effects of Infant Nutrition on Cholesterol Synthesis Rates
}

\author{
MARIA LOURDES A. CRUZ, WILLIAM W. WONG. FRANCIS MIMOUNI, DAVID L. HACHEY, \\ KENNETH D. R. SETCHELL, PETER D. KLEIN, AND REGINALD C. TSANG \\ Perinatal Research Institute. Lniversity of Cincinnati/Children's Hospital Medical Conter. (incinnati. Ohio

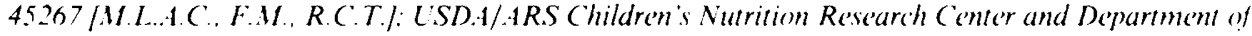 \\ Pediatrics. Baylor College of Medicine. Houston. Texas 77030 /W.W.W., D.L.H., P.D.K.l: and Mass \\ Spectrometry Laboratory: Division of Gastroemerology, Children's Hospital Hedical Center.
} (incinnati, Ohio $4.229 /$ K.D.R.S.J

\begin{abstract}
Nutrient effects on cholesterol fractional synthesis rates (FSR) in infancy by stable isotope determination have not been studied. We hypothesized that FSR is significantly reduced with high dietary cholesterol and phytoestrogen intake and increased with low dietary cholesterol and phytoestrogen intake. We prospectively studied 33 term male infants exclusively fed human milk (high cholesterol, low phytoestrogen, $n=12$ ), cow milk-based formula (low cholesterol, low phytoestrogen, $n=8$ ), soy milk-based formula (zero cholesterol, high phytoestrogen, $n=7$, or soy milk-based formula modified to contain cholesterol (low cholesterol, high phytoestrogen, $n=6$ ) during the first 4 mo of life. Cholesterol FSR was determined from rate of incorporation of deuterium into erythrocyte membrane cholesterol, and urinary isoflavone excretion (an index of dietary phytoestrogen exposure) was measured by gas chromatography-mass spectrometry. Significant differences in cholesterol FSR were found. FSR $(\% / d)$ was lowest in human milk $(2.62 \pm 0.38)$, highest in soy milk-based formula $(9.40 \pm 0.51)$, and intermediate in cow milk-based and modified soy milk-based formula $(6.90 \pm 0.48$ and $8.03 \pm 0.28$, respectively), $p<0.0001$. Cholesterol FSR was significantly lower in modified soy milk-based compared with soy milk-based formula, $p<$ 0.05 . We also show for the first time that dietary phytoestrogens are absorbed and excreted by the infant fed soy protein-based formula. Urinary isoflavone excretion was inversely related to cholesterol FSR, but it was not significantly related to serum cholesterol concentration. We conclude that the type of infant nutrition and dietary cholesterol are major factors influencing cholesterol fractional synthesis rates in infancy. (Pediatr Res 35: 135-140, 1994)
\end{abstract}

\section{Abbreviations}

FSR, fractional synthesis rate

HUM, human milk

COW, cow milk-based formula

SOY, soy milk-based formula

MSOY, modified soy milk-based formula

Received April 8, 1992; accepted September 22, 1993.

Correspondence: Maria Lourdes Cruz. M.D., Division of Neonatology, Department of Pediatrics, University of Cincinnati, 231 Bethesda Ave. (ML 541). Cincinnati, OH 45267-0541.

Supported in part by NIH HD 07200 Perinatology Training Grant, NIH Clinical Research Center Grant RR00068. NIH HD 20748. Perinatal Research Institute. and USDA/ARS 58-7MN1-6-100. Formula supplied by Ross Laboratories.
In adults, a high cholesterol intake inhibits endogenous cholesterol synthesis (1). However, the influence of nutrition on cholesterol metabolism in early life is largely unstudied. HUM contains a relatively large concentration of dietary cholesterol $(2.59-3.88 \mathrm{mmol} / \mathrm{L})$. By contrast. COW have low concentrations of cholesterol $(0.28-0.85 \mathrm{mmol} / \mathrm{L})$, and SOY contain no cholesterol. Differences in dietary cholesterol intake by infants may affect endogenous cholesterol FSR, and this rate may have relevance to cholesterol metabolism later in life $(2,3)$. To our knowledge, no studies have been published on the effects of a diet completely devoid of cholesterol on endogenous cholesterol synthesis in human beings. The cholesterol-lowering effect of soy protein foods, although not well understood, is well established in infants and adults. Soy protein contains significant amounts of nonsteroidal estrogen analogues of the isoflavone class $(4,5)$ and it has been suggested that ingestion of these phytoestrogens may also play a role in cholesterol homeostasis (4).

A few studies of sterol balance in small $(n=5-10)$ and heterogenous (3-19 mo old) populations (6-9) have been conducted in infants. However, no in vivo studies have been conducted on cholesterol synthesis rates using stable isotope techniques in a homogenous human infant population. The relationships between dietary cholesterol, phytoestrogen intake, serum cholesterol concentration, and endogenous cholesterol synthesis rates in infants have not been previously studied.

We therefore conducted a prospective, partially randomized, controlled study evaluating the effects of infant nutrition on cholesterol synthesis rates to determine whether cholesterol synthesis rates are influenced by cholesterol intake. We hypothesized that cholesterol synthesis rates will be negatively correlated with dietary cholesterol intake: thus. FSR will be lowest in infants fed HUM, highest in infants fed SOY, and intermediate in infants fed COW. A fourth group of infants fed MSOY, formulated to contain the same amount of cholesterol as COW, was included to determine whether the cholesterol component could be specifically responsible for alterations in cholesterol synthesis rates. As an ancillary hypothesis, we theorized that the high phytoestrogen content of SOY and MSOY (reflected by urinary isoflavone excretion) will contribute to an inhibition of cholesterol synthesis and, therefore, lower serum cholesterol concentrations in infants fed these formulas.

\section{MATERIALS AND METHODS}

Study population. Thirty-nine healthy Caucasian male infants who were full term, appropriate for gestational age, and had no known parental history of hypercholesterolemia or hypertriglyceridemia were recruited from the normal newborn nurseries of the University Hospital of Cincinnati and other area hospitals. Thirteen infants exclusively breastfed for the first 4 mo of life 
comprised the HUM-fed group. Twenty-six infants comprised the formula groups. Twenty of these infants were randomized to receive either COW (Similac with Iron, $20 \mathrm{cal} /$ ounce; Ross Laboratories, Columbus, $\mathrm{OH})(n=10)$ or SOY (Isomil with Iron, $20 \mathrm{cal} /$ ounce; Ross Laboratories, Columbus, $\mathrm{OH})(n=10)$. The last six formula-fed infants were assigned to receive MSOY, specially formulated to contain a cholesterol content $(0.28$ $\mathrm{mmol} / \mathrm{L}$ ) similar to that of COW. Assignment (rather than randomization) was due to initial delay in the availability of modified formula. The compositions of the different formulas are summarized in Table 1 . All infants received their assigned formula within the first 3 to $7 \mathrm{~d}$ of life. Formula was provided free to the formula-fed infants for the entire duration of the study to ensure compliance. The study was approved by the institutional review boards of all the involved hospitals, and informed consent was obtained before enrollment of the infants.

Thirty-three infants completed the study (HUM, $n=12$; COW, $n=8$; SOY, $n=7$; MSOY, $n=6$ ). One infant from the HUM group was eliminated from the study because the mother had stopped breastfeeding within $4 \mathrm{wk}$; two formula-fed infants (one from the COW group and one from the SOY group) were excluded from the study because of formula intolerance; and one infant from the COW group and two infants from the SOY group were lost to follow-up.

Mothers from the four groups were required to keep 3-d diet records for each month until the testing period at $4 \mathrm{mo}$. Mothers recorded information regarding the frequency of breastfeeding, the volume of supplemental COW given per day (Similac with Iron), and the volume of formula per day in the formula groups. By design, other forms of nutrition such as cereal were not allowed (except for multivitamins).

Cholesterol synthesis rates and urinary isoflavone excretion were determined at 4 mo of age. The age 4 mo was chosen because infants in the first 4 mo of life generally received exclusively human milk or formula (10), so little potential existed for interference from other diets. Thus, differences in FSR, lipid profiles, and urinary isoflavone excretion could be specifically attributed to the type of milk consumed. Measurements were performed over a 4-d period. On d 1, a urine sample (approximately $15 \mathrm{~mL}$ ) was obtained to determine baseline body water deuterium enrichment and urinary isoflavone concentrations. The infant was then orally given $500 \mathrm{mg} / \mathrm{kg}$ body weight of deuterium oxide $\left(\mathrm{D}_{2} \mathrm{O}, 99.96 \%\right.$ deuterium; Isotech Inc., Miamisburg, $\mathrm{OH}$ ). On $\mathrm{d} 2$ and 3, a blood sample for deuterium excess enrichment was obtained, after which $50 \mathrm{mg} / \mathrm{kg}$ body weight of $\mathrm{D}_{2} \mathrm{O}$ was given to maintain constant body water $\mathrm{D}_{2} \mathrm{O}$ enrichment. On d 4, a final blood sample was obtained. No fasting period was required before phlebotomy, and all blood samples (8 $\mathrm{mL}$ each day on $\mathrm{d} 2-4)$ were drawn between $0900 \mathrm{~h}$ and 1130 h.

Laboratory methods. Determinations of serum cholesterol and lipid profile were performed at the Medical Research Laboratories in Cincinnati, $\mathrm{OH}$, with enzymatic techniques validated by the National Institutes of Health Lipid Research Clinics. Serum LDL cholesterol concentrations were calculated from serum total

Table 1. Composition of types of milk used in study*

\begin{tabular}{lcccc}
\hline & HUM & COW & SOY & MSOY \\
\hline Calories & 680 & 676 & 676 & 690 \\
Protein $(\mathrm{g})$ & 10.5 & 15 & 18 & 19.8 \\
Carbohydrate (g) & 72 & 72.3 & 68.3 & 67.7 \\
Fat (g) & 39 & 36.3 & 36.9 & 37.8 \\
Polyunsaturated/satu- & 0.3 & 0.8 & 0.8 & 0.8 \\
$\quad$ rated ratio & & & & \\
Cholesterol (mmol) & $2.59-3.88$ & $0.28-0.85$ & 0 & 0.28 \\
Linoleic acid (mg) & 3971 & 8790 & 8790 & 8790 \\
\hline
\end{tabular}

* Composition of HUM varies with stage of lactation and among mothers. Composition of formulas based on manufacturer's estimates. Numbers are per liter. cholesterol concentrations using the equation formulated by Friedwald et al. (11).

Determinations of cholesterol FSR were performed by the Stable Isotope Laboratory at the Children's Nutrition Research Center in Houston, Texas, using the methodologic procedure developed by Wong et al. (12). FSR was determined from the rate of incorporation of deuterium into red blood cell membrane cholesterol. Briefly, the analytical procedure for FSR involves lipid extraction, purification of erythrocyte-free cholesterol by HPLC, conversion of cholesterol to carbon dioxide and water by combustion, reduction of the latter to hydrogen, and, finally, analysis of deuterium enrichment using gas/isotope ratio mass spectrometry (12). To calculate FSR, an exponential model using CONSAM, a compartmental modeling program from the $\mathrm{Na}$ tional Institutes of Health (12a), was used, with the following formula:

$$
\text { Enrichment }=0.4783 \times \mathrm{E}_{\mathrm{BW}}\left(1-\mathrm{e}^{-\mathrm{K} \times \mathrm{T}}\right)
$$

where $E_{B W}$ is the deuterium enrichment in body water, $K$ is the FSR in \%/day, and $\mathrm{T}$ is the time in days.

Mean baseline deuterium enrichment in erythrocyte membrane cholesterol determined in 13 other normal infants from Cincinnati was used for the baseline value $(0.01184 \pm 0.004$ atom \%). The constant 0.4783 is the fraction of hydrogen atoms incorporated into the cholesterol molecule (22/46) from body water. The coefficient of variation by this method ranged from 11 to $50 \%$; higher coefficients of variation occur at low FSR as a result of reduced precision in isotope measurement because of minimal incorporation of deuterium into cholesterol from body water.

Urinary isoflavone excretion, specifically equol, daidzein, genistein, and total isoflavones (calculated as the sum of the three metabolites), were determined by mass spectrometry using previously described techniques $(4,5)$. Isoflavones and their conjugates were quantitatively extracted from urine by liquid-solid extraction, isolated by lipophilic gel chromatography before conversion to the volatile trimethylsilyl ethers, and analyzed by selected ion monitoring gas chromatography-mass spectrometry.

Statistical analysis. The statistical analysis system of Macintosh JMP, developed by the SAS Institute, Inc. (Cary, NC) was used for data management and analysis. One-way analysis of variance was used to test differences between groups. When values were normally distributed (lipid profiles and FSR), TukeyKramer method for multiple comparisons was used to determine differences between pairs of groups. Results for urinary isoflavones were log-transformed to approximate a gaussian distribution before testing for differences between groups. General linear models were used to determine correlations between the outcome variable (FSR) and the independent variables. A nonparametric test for ordered alternatives was used to determine correlations between quantity of cholesterol intake and FSR (13). Statistical significance was considered for $p$ values less than 0.05 .

\section{RESULTS}

Results are presented as mean \pm SEM. Demographic data are summarized in Table 2. No significant differences were seen in birth weight and weight, length, and ponderal index at 4 mo among groups. No significant differences were observed in volume of milk intake among the formula groups as determined from dietary records.

Serum cholesterol and LDL cholesterol concentrations were highest in HUM, intermediate in COW and MSOY, and lowest in SOY, and were significantly different among groups (Table 3). No differences were observed in serum HDL cholesterol and triglyceride concentrations among groups.

Deuterium enrichment of body water and erythrocyte cholesterol are shown in Figure 1. No difference was seen in deuterium enrichment in body water among groups. Cumulative erythrocyte cholesterol enrichment was consistently lowest in HUM, 
Table 2. Characteristics of study population*

\begin{tabular}{cc}
$\begin{array}{c}\text { Type of } \\
\text { milk }\end{array}$ & $\begin{array}{c}\text { Birth } \\
\text { weight }(\mathrm{kg})\end{array}$ \\
\hline HUM & $3.6 \pm 0.1$ \\
COW & $3.5 \pm 0.1$ \\
SOY & $3.3 \pm 0.1$ \\
MSOY & $3.7 \pm 0.1$
\end{tabular}

NS

$$
\begin{gathered}
\text { Ponderal index } \\
\left(\frac{\text { weight }(\mathrm{g}) \times 100}{\text { length }(\mathrm{cm})^{3}}\right) \\
2.59 \pm 0.09 \\
2.47 \pm 0.13 \\
2.65 \pm 0.13 \\
2.44 \pm 0.13 \\
\mathrm{NS}
\end{gathered}
$$

\begin{tabular}{|c|c|c|c|c|c|}
\hline $\begin{array}{c}\text { Type of } \\
\text { milk }\end{array}$ & $\begin{array}{c}\text { Chol } \\
(\mathrm{mmol} / \mathrm{L})\end{array}$ & $\begin{array}{c}\text { Trig } \\
(\mathrm{mmol} / \mathrm{L})\end{array}$ & $\begin{array}{c}\text { HDL-c } \\
(\mathrm{mmol} / \mathrm{L})\end{array}$ & $\begin{array}{c}\text { LDL-c } \\
(\mathrm{mmol} / \mathrm{L})\end{array}$ & $\operatorname{FSR}\left({ }^{\circ} c / d\right)$ \\
\hline HUM & $4.47 \pm 0.20 \dagger$ & $1.30 \pm 0.20$ & $1.32 \pm 0.09$ & $2.54 \pm 0.21 \ddagger$ & $2.97 \pm 0.49 \$$ \\
\hline cow & $3.34 \pm 0.24$ & $1.44 \pm 0.25$ & $1.22 \pm 11$ & $1.45 \pm 0.25$ & $9.43 \pm 1.12$ \\
\hline SOY & $3.11 \pm 0.26$ & $1.38 \pm 0.27$ & $1.43 \pm 0.12$ & $0.99 \pm 0.27$ & $12.02 \pm 1.36$ \\
\hline MSOY & $3.40 \pm 0.28$ & $1.39 \pm 0.29$ & $1.26 \pm 0.13$ & $1.49 \pm 0.29$ & $10.55 \pm 1.05$ \\
\hline
\end{tabular}

Formula intake
(ounces/d)
Less than 1
27.4
26.8
27.9
NS +

* Mean \pm SEM.

+ NS among the formula groups.

Table 3. Serum lipid profiles and FSR determination.**

* Mean \pm SEM. Chol. Cholesterol: Trig, triglycerides; HDL-c. HDL cholesterol; LDL-c, low LDL cholesterol.

+ HUM is COW, $p=0.0067$; HUM is SOY, $p=0.002$; HUM is MSOY, $p=0.01$. No significant difference in serum cholesterol concentrations was found among the formula groups.

$\ddagger$ HUM s COW, $p=0.01$ : HUM is SOY, $p=0.001$ : HUM is MSOY, $p=0.03$. No significant difference in serum L.DL cholesterol concentrations was found among the formula groups.

$\S$ HUM is COW, SOY, or MSOY, $p<0.0001$ : COW is SOY, $p=0.0066$ : COW $w$ MSOY, $p=0.1$ : SOY $w$ MSOY, $p=0.03$.

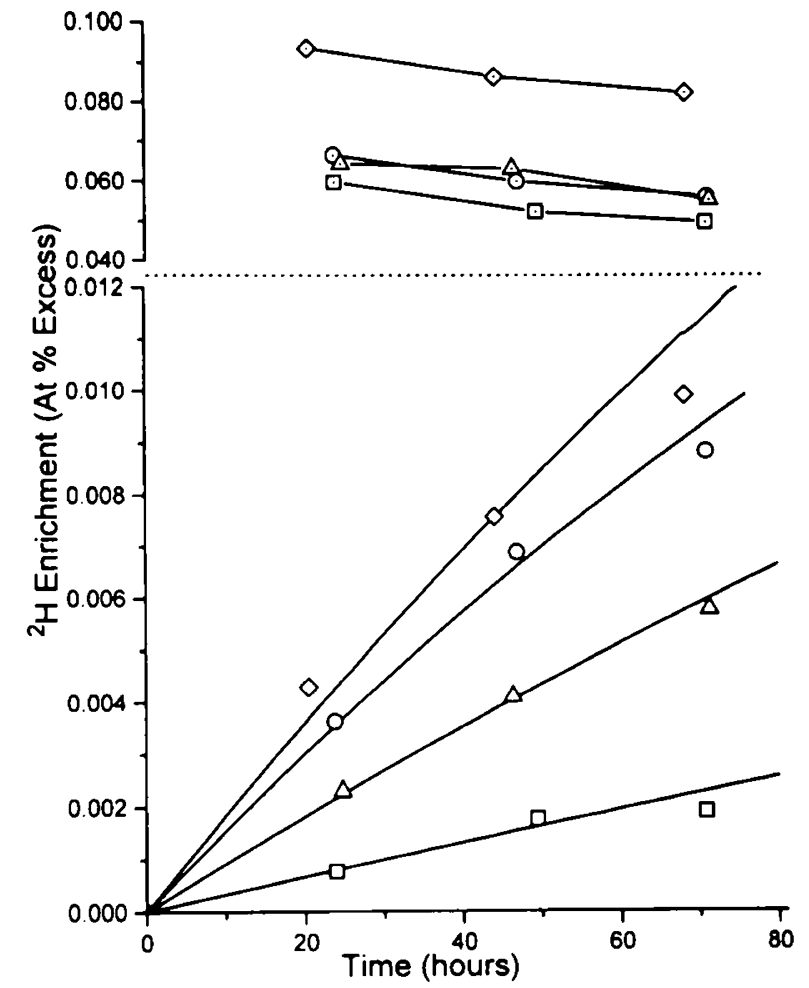

Fig. 1. Deuterium enrichment of body water (top panel) and erythrocyte membrane cholesterol ( $b o t t o m$ panel) over time for a single subject in each feeding group who had the median value for the cholesterol fractional synthesis rate. Deuterium enrichment is shown for HUM ( $\square$ ). $\operatorname{COW}(\triangle)$, SOY $(O)$, and MSOY () in both panels. The solid lines on the lower panel are the fitted lines from the exponential model. The mean deuterium enrichment in body water was similar among groups: range, $0.026-0.093$ atom \% excess. intermediate in COW and MSOY, and highest in SOY over the 4-d study period.

Differences in FSR among the four groups were highly significant (Table 3). FSR of HUM was significantly lower compared with all other groups. FSR of SOY was significantly higher than that of COW and MSOY, whereas FSR did not differ significantly between COW and MSOY. By nonparametric test for ordered alternatives, quantity of dietary cholesterol intake was significantly and negatively associated with FSR $(p=0.0001)$. Correlation and $p$ values were unchanged even when data from four infants (three from HUM and one from COW) with high serum cholesterol and triglyceride concentrations were excluded from the analysis. Combining all groups, a significant negative correlation existed between serum total cholesterol concentration and FSR $(r=-0.59, p<0.005)$, and between serum LDL-cholesterol concentrations and FSR $(r=-0.67, p<0.005)$ (Fig. 2).

Total and individual urinary isoflavone excretion for 31 infants are presented in Figure 3. Equol excretion was similar among the groups. However, urinary daidzein, genistein, and total isoflavone excretion differed significantly among groups, being low in HUM and COW and high in SOY and MSOY. To estimate the effect of dietary phytoestrogens on cholesterol FSR. we used a multiple regression analysis model controlling for group effect (because groups differed not only in quantity of dietary phytoestrogen but also in quantity of dietary cholesterol). Applying this model, we found that FSR was negatively associated with total urinary isoflavone excretion, $p=0.05$. Serum cholesterol concentrations were not significantly associated with urinary isoflavone excretion.

\section{DISCUSSION}

To our knowledge, only four previous cholesterol balance studies related to diet in infants have been reported. Using traditional balance techniques, researchers have estimated that endogenous cholesterol synthesis ranges from 0 to $40 \mathrm{mg} / \mathrm{kg} / \mathrm{d}$ in infants on various diets (6-9). Conclusions from these studies may be limited by small sample size, implementation in nonhomogenous populations, and problems associated with balance studies, such as dependence on completeness of stool collection.

In our study, we minimized some of these problems by con- 


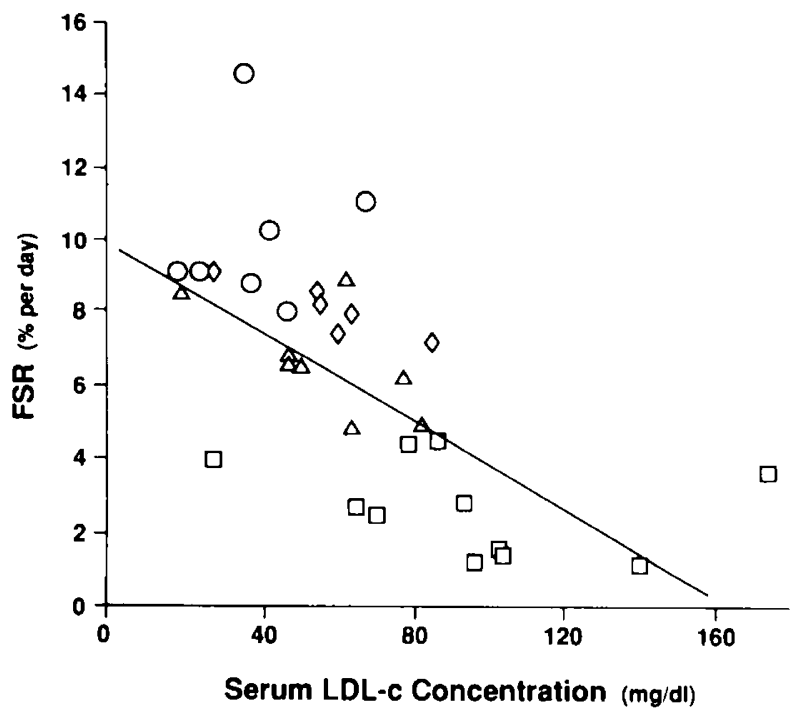

Fig. 2. Cholesterol FSR vs serum LDL cholesterol ( $L D L-c)$ concentrations. FSR, in \%/d, are plotted on the y-axis. Serum LDL-cholesterol concentrations, in $\mathrm{mg} / \mathrm{dL}$, are plotted on the $\mathrm{x}$-axis. HUM, O: COW, $\triangle$ : SOY, $\square$; and MSOY, $\therefore$ A significant negative correlation was found between FSR and serum LDL-cholesterol concentration, with $r$ of -0.67 at $p=0.003$

ducting the study prospectively and in a well-controlled population of 4-mo-old Caucasian males fed four distinct diets. Furthermore, endogenous cholesterol synthesis rates were more specifically determined with stable isotopes by measuring the incorporation of deuterium into erythrocyte membrane cholesterol.

Endogenous cholesterol synthesis determinations based on deuterium incorporation was first described by Rittenberg and Schoenheimer (14) and later modified by others $(12,15)$. The methodologic procedure is based on previous studies using tritiated water (16-18), and the interpretation of results depends on three assumptions (19). First, a constant fraction of the deuterium atoms in newly synthesized cholesterol is derived from deuterium in the body water pool. Because water freely and rapidly diffuses across cell membranes, plasma water deuterium concentration provides a measure of precursor enrichment, and deuterium enrichment of the newly synthesized cholesterol molecules corresponds to enrichment of the central pool (M1), which includes the liver, plasma, and intestine (20). Second, within the central pool, rapid movement of newly synthesized cholesterol from the liver to plasma lipoproteins and then to cell membranes occurs, and therefore the deuterium content of erythrocyte membranes reflects newly synthesized cholesterol (21). Third, deuterium uptake by cell membranes represents synthesis from the central pool only, and it does not represent influx of newly formed cholesterol from other pools or synthesis by erythrocytes. On the basis of these assumptions, it is believed that the stable isotope method provides a more reliable measurement of cholesterol synthesis compared with traditional balance techniques (17).

Our group has modified and adapted the methodologic procedure for use in human infants by determining FSR using erythrocyte membrane cholesterol (rather than plasma free cholesterol). The validity of determining cholesterol synthesis rates in erythrocytes rather than from plasma cholesterol is supported by our preliminary studies wherein we performed extensive kinetic simulations of the central pool defined by other investigators $(20,22)$. Results of these studies indicate that any of the components of the central pool (e.g. plasma or red cell membrane) may serve as valid surrogates for the whole pool (unpublished observations). Additionally, it has been recently demonstrated in human adults that by $24 \mathrm{~h}$ after deuterium loading,

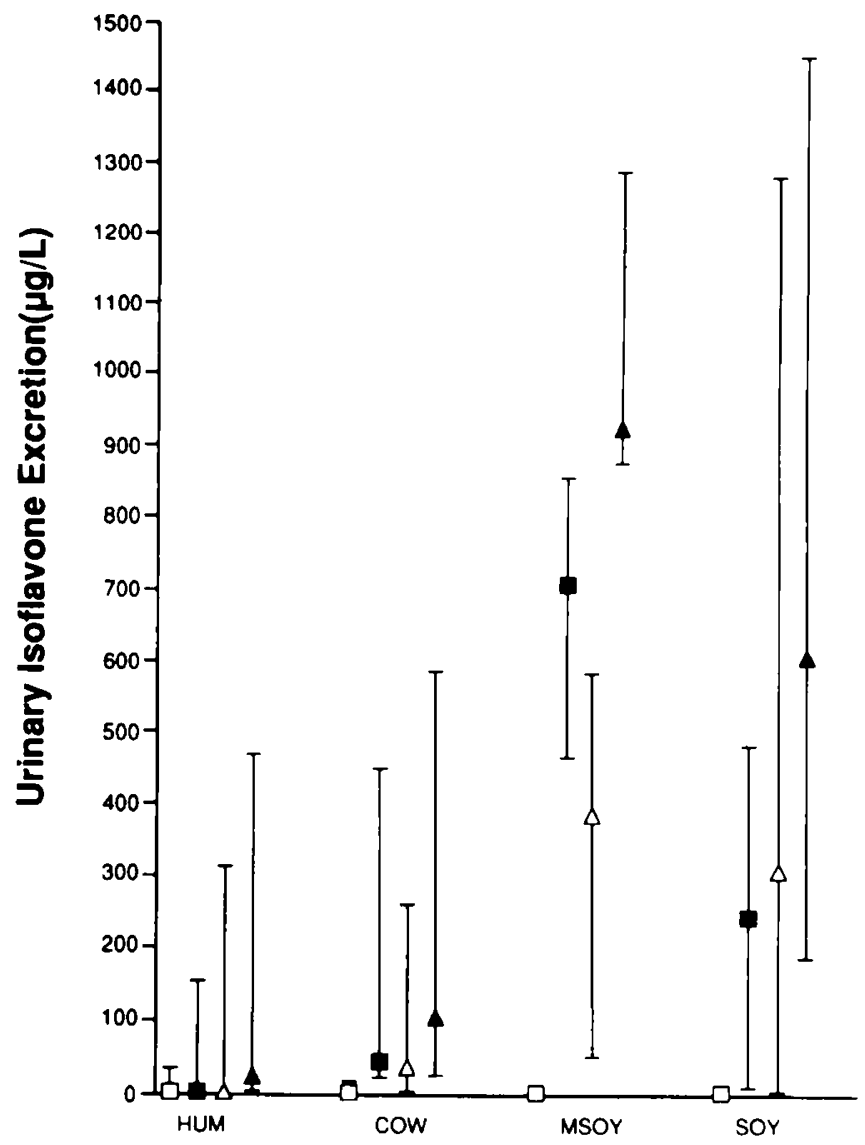

Fig. 3. Median and range of individual and total urinary isoflavones in each group. Urinary isoflavones, in $\mu \mathrm{g} / \mathrm{L}$, are plotted on the $y$-axis. The lowest and highest values are delineated by the lower and upper limits of each line; median values are represented by boxes or triangles. The different groups are plotted on the $\mathrm{x}$-axis. Equol, $\square$ : daidzein, genistein, $\Delta$; and total urinary isoflavones, $\Delta$. Equal excretion was similar among groups. For all intergroup comparisons for daidzein, $p<0.05$. except for COW is SOY and SOY vs MSOY, where value was NS. All intergroup comparisons for genistein were NS, except for HUM vs SOY or MSOY, where $p<0.05$. For all intergroup comparisons for total urinary isoflavones, $p<0.05$, except for HUM $v s$ COW and SOY is MSOY, where value was NS.

cholesterol synthesis rates calculated from deuterium uptake by erythrocyte membrane cholesterol are similar to those calculated from plasma cholesterol enrichment (23). Consequently, we have been able to use relatively small volumes of blood $(8 \mathrm{~mL}$ every $24 \mathrm{~h}$ for $3 \mathrm{~d}$ compared with 20 to $120 \mathrm{~mL}$ every $24 \mathrm{~h}$ for $2 \mathrm{~d}$ ). Thus, in this study, FSR is expressed as the rate of entry of deuterium into erythrocyte membrane cholesterol during the study period, calculated using the equation given earlier.

Another factor that may affect FSR determinations is differences in the natural abundance of deuterium within erythrocytes based on diet, age, and geographic location. In this study, baseline blood samples were not directly collected from study infants so that the number of blood draws and the amount of blood taken from these infants would be minimized. Rather, we measured the natural abundance of deuterium in erythrocyte cholesterol in 13 other 4 - to 6-mo-old normal infants in the Cincinnati area. The results were as follows: all infants, $0.0118 \pm 0.0004$ atom $\%$; three infants on HUM, $0.0117 \pm 0.0002$ atom \%; five infants on COW, $0.0117 \pm 0.0002$ atom \%; and five infants on SOY, $0.0120 \pm 0.0005$ atom $\%$. Individual baseline natural abundance determinations for each infant would be ideal, but limitations on blood sampling necessitated this approach.

Plasma water deuterium enrichment was used to compute an index of cholesterol precursor enrichment in the central pool for 
each subject. The observed variability in body water deuterium enrichment among patients (range. 9.0259-0.0928 atom \%), would not affect calculations because it is relatively constant within a subject $( \pm 7 \%)$. Also, because the range of variation among the four groups was similar during the whole study period and because the plateau body water deuterium enrichment for each infant was relatively constant $( \pm 7 \%)$, the probability that these minor variations in daily deuterium enrichment would significantly affect FSR calculations is relatively low.

It may be argued that the lower FSR in the HUM group is an artifact resulting from a "dilution" effect. High dietary cholesterol intakes and plasma cholesterol concentrations of HUM-fed infants may conceivably lead to higher cholesterol content in the central pool and subsequently create a "dilution" effect on calculations of synthesis rates. This cannot be the case, however, because the central pool of HUM-fed infants is probably about 15 to $20 \%$ larger compared with formula-fed infants, based on previous piglet studies (24). Additionally, erythrocyte membrane cholesterol content is fairly constant regardless of diet. as demonstrated in adults $(12,22)$ and in infants fed either HUM or formula $(25,26)$. The $15-20 \%$ difference in central pool between HUM-fed and formula-fed groups and the $30 \%$ difference in plasma cholesterol concentrations are therefore neither sufficient to explain nor proportionate to the almost 4 -fold difference in cholesterol synthesis rates among groups.

The results of our study indicate that adaptive regulatory mechanisms in early infancy enable human infants to respond to differences in cholesterol intakes. Teleologically, such homeostatic mechanisms would prevent excess cholesterol accumulation during high cholesterol intakes or, conversely, provide for an increase in cholesterol availability during instances of low or negligible intake. If we assume that HUM is the "gold standard" for infant nutrition and values for FSR in HUM-fed infants are considered to be the "norm," these data would indicate that COW-fed infants have a 2.6-fold increase and SOY-fed infants have a 3.7-fold increase in FSR. We suggest that the apparent need for cholesterol in early infancy, a period of rapid growth, is reflected by the marked increase in cholesterol synthesis rates of the SOY-fed infants. The distinct differences in FSR in the present study may theoretically be of value when the infant is "challenged" with high cholesterol intake later in life $(2,27,28)$.

It has been previously suggested that the cholesterol-lowering effect of soy protein may in part be attributed to the presence of an abundance of nonsteroidal estrogens of the isoflavone class (4) that are a natural constituent of soy-based products, including SOY (5). These isoflavones are metabolized further in the gastrointestinal tract, absorbed, undergo an enterohepatic circulation, and excreted in urine in common with many endogenous steroids $(29,30)$. Urinary excretion of the isoflavones therefore reflects phytoestrogen intake. This study provides the first evidence that isoflavones present in SOY (i.e. daidzein and genistein) are absorbed from the gastrointestinal tract and excreted in relatively large amounts in the urine of SOY-fed infants (Fig. 3 ). The absence of equol in the urine of SOY-fed infants is presumed to be the result of the developmental lack of microflora required for bacterial metabolism of the precursors, daidzein and genistein.

Estrogens appear to play a role in cholesterol homeostasis (31, 32). Isoflavones are weak estrogens, and therefore sufficient dietary intake may potentially affect cholesterol metabolism. Our data are consistent with the thesis that the phytoestrogen content of SOY may be sufficient to influence cholesterol metabolism by decreasing cholesterol FSR; however, we could not demonstrate any effect on serum cholesterol concentration in this study. The inverse relationship between cholesterol FSR and isoflavone exposure appears partially concealed by the overriding influence of cholesterol intake.

Other factors that may influence cholesterol homeostasis include differences in the polyunsaturated/saturated ratio (33) and protein composition (34). This study design did not allow for testing of the specific influence of the polyunsaturated/saturated ratio or protein composition on cholesterol FSR

We have examined for the first time the in vivo endogenous cholesterol synthesis rates of human infants and have established that cholesterol synthesis rates are lowest in infants with the highest cholesterol intake, intermediate in infants with moderate cholesterol intake, and highest in infants with no cholesterol intake. These data also establish for the first time that dietary phytoestrogens are absorbed and excreted by the human infant fed SOY and that a negative association with cholesterol synthesis rates exists.

We conclude that the type of infant nutrition significantly affects serum lipid profiles, cholesterol synthesis rates, and urinary isoflavone excretion. We have demonstrated that the human infant is able to respond to different quantities of dietary cholesterol intakes and that this response is a major factor influencing cholesterol synthesis rates.

Acknowledgments. The authors thank Debbie Donovan, R.N.. who coordinated the clinical study: Jeffrey Leggitt. Lucinda Clarke, and Marisol Llaurador for performing the FSR assays; and James Carlson for performing the urinary isoflavone determinations.

\section{REFERENCES}

1. Klein PD, Jiang J, Wong WW, Hachey DL, Insull W 1990 In vivo demonstration of cholesterol synthesis inhibition by cholesterol feeding in man. Am J Clin Nutr 51:516(abstr)

2. Mott GE. Jackson EM, McMahan CA. McGill H 1990 Cholesterol metabolism in adult baboons is influenced by infant diet. $J$ Nutr 120:243-251

3. Marmot MG. Page C 1980 Effect of breastfeeding on plasma cholesterol and weight in young women. J Epidemiol Community Health 34:164-167

4. Setchell KDR 1985 Naturally occurring non-steroidal estrogens of dietary origin. In: McLachlan JA (ed) Estrogens in the Environment. Elsevier Science, New York, pp 69-86

5. Setchell KDR. Welsh MB, Lim C 1987 High performance liquid chromatographic analysis of phytoestrogens in soy protein preparations with ultraviolet, electrochemical, and thermospray mass spectrometry. J Chromatogr 386:315-323

6. Gamble JL, Blackfan K 1920 Evidence indicating a synthesis of cholesterol by infants. J Biol Chem 42:401-409

7. Huang CTL. Rodriguez JT, Woodward WE, Nichols B 1976 Comparison of patterns of fecal bile acid and neutral sterol between children and adults. Am J Clin Nutr 29:1196-1203

8. Potter JM. Nestel P 1976 Greater bile acid excretion with soy bean than with cow milk in infants. Am J Clin Nutr 29:546-551

9. Nestel PJ, Pyser A, Boulton T 1979 Changes in cholesterol metabolism in infants in response to dietary cholesterol and fat. Am J Clin Nutr 32:21772182

10. Purvis GA, Bartholmey S 1988 Infant feeding practices: commercially prepared baby foods. In: Tsang RC. Nichols BL (eds) Nutrition During Infancy. Hanley Belfus, Philadelphia, pp 399-417

11. Friedwald RT, Levy RI, Fredickson P 1972 Estimation of the concentration of low density lipoprotein concentration without use of preparative ultracentrifuge. Clin Chem 18:499-502

12. Wong WW, Hachey D. Feste A. Leggitt J. Clarke L. Pond W. Klein P 1991 Measurement of in vivo cholesterol synthesis from ${ }^{2} \mathrm{H}_{2} \mathrm{O}$ : a rapid procedure for the isolation, combustion, and isotopic assay of erythrocyte cholesterol. J Lipid Res 32:1049-1056

12a. Boston RC, Greif PC, Berman M 1981 Conversational SAAM: an interactive program for kinetic analysis of biological systems. Comp Prog Biomed 13:111-119

13. Hollander M, Wolfe D 1973 Nonparametric Statistical Methods. Wiley, New York, pp 120-123

14. Rittenberg D, Schoenheimer R 1937 Deuterium as an indicator in the study of intermediary metabolism. XI. Further studies on the biological uptake of deuterium into organic substances, with special reference to fat and cholesterol formation. J Biol Chem 121:235-253

15. Jones PJH, Scanu Am, Schoeller D 1988 Plasma cholesterol synthesis using deuterated water in humans: effect of short-term food restriction. J Lab Clin Med 111:627-633

16. Andersen JM. Dietschy J 1979 Absolute rates of cholesterol synthesis in extrahepatic tissues measured with ${ }^{3} \mathrm{H}$-labeled water and ${ }^{14} \mathrm{C}$-labeled substrates. J Lipid Res 20:740-752

17. Dietschy JM. Spady D 1984 Measurement of rates of cholesterol synthesis using tritiated water. J Lipid Res 25:1469-1476

18. Esterman AL, Cohen BI, Javitt N 1985 Cholesterol metabolism: use of $\mathrm{D}_{2} \mathrm{O}$ for determination of synthesis rate in cell culture. J Lipid Res 26:950-954

19. Jones PJ 1990 Use of deuterated water for measurement of short-term cholesterol synthesis in humans. Can J Physiol Pharmacol 68:955-959 
20. Dell RB, Mott GE, Jackson EM, Ramakrishnan R, Carey KE, McGill HC, Goodman D 1985 Whole body and tissue cholesterol turnover in the baboon. J Lipid Res 26:327-337

21. London IM. Schwarz H 1953 Erythrocyte metabolism. The metabolic behavior of the cholesterol of human erythrocytes. J Clin Invest 32:1248-1252

22. Schwartz CC, Vlahcevic ZR, Berman M, Meadows J 1965 Central role of high density lipoprotein in plasma free cholesterol metabolism. J Clin Inves 70:105-116

23. Li Z-C, Jones PJ, Connor W 1992 Comparison of deuterium uptake kinetics between plasma and erythrocyte cholesterol pools. 35th Annu Meeting Proc Can Fed Biol Soc 93(abstr)

24. Hachey DL. Wong WW, Pond W, Clark L, Llaurador M, Klein P 1992 Cholesterol (C) synthesis in genetically lean and obese piglets on either low or high C diets. FASEB J 6:1081(abstr)

25. De Lucchi C. Pita ML. Periago JL. Gil A 1988 Influences of diet and postnatal age on the lipid composition of red blood cell membrane in newborn infants. Ann Nutr Metab 32:231-239

26. Putnam JC. Carlson SE, DeVoe PW, Barness L 1982 The effect of variations in dietary fatty acids on the fatty acid composition of erythrocyte phospha. tidylcholine and phosphatidylethanolamine in human infants. Am J Clin Nutr 36:106-114

27. Reiser R, Sidelman J 1972 Control of cholesterol homeostasis by cholesterol in the milk of the suckling rat. J Nutr 102:1009-1016
28. Mott GE, Jackson EM. McMahan CA. Farley CM, McGill H 1985 Cholesterol metabolism in juvenile baboons: influence of infant and juvenile diets. Arteriosclerosis 5:347-354

29. Setchell KDR. Borriello SP. Hulme P, Kirk DN. Axelson M 1984 Nonsteroidal estrogens of dietary origin: possible roles in hormone-dependent disease. Am $\mathrm{J}$ Clin Nutr 40:569-578

30. Setchell KDR, Adlercreutz H 1988 Mammalian lignans and phytoestrogens: recent studies on their formation, metabolism and biological role in health and disease. In: Rowland IA (ed) The Role of Gut Microflora in Toxicity and Cancer. Academic Press, London. pp 315-345

31. Mukherjee S. Brose A 1968 Studies on estrogen regulation on cholesterol biosynthesis in rat liver microsomes. Biochim Biophys Acta 164:357-368

32. Subbiah MTR 1977 Effect of long term administration of estrogens on the subcellular distribution of cholesterol and the activity of rate-limiting enzymes in cholesterol biosynthesis and degradation in pigeon liver. Endokrinologie 70:257-262

33. Spady DK, Dietschy JM 1988 Interaction of dietary cholesterol and triglycerides in the regulation of hepatic low density lipoprotein transport in the hamster. J Clin Invest 81:300-309

34. Nagata Y, Ishiwaki N. Sugano M 1982 Studies on the mechanism of antihvpercholesterolemic action of soy protein and soy protein type amino acid mixtures in relation to casein counterparts in rats. J Nutr 112:1614-1625 